\title{
The Effects of Fractions from Shiitake Mushroom on Composition and Cariogenicity of Dental Plaque Microcosms in an In Vitro Caries Model
}

\author{
Egija Zaura, ${ }^{1}$ Mark J. Buijs, ${ }^{1}$ Michel A. Hoogenkamp, ${ }^{1}$ Lena Ciric, ${ }^{2}$ Adele Papetti, ${ }^{3}$ \\ Caterina Signoretto, ${ }^{4}$ Monica Stauder, ${ }^{5}$ Peter Lingström, ${ }^{6}$ Jonathan Pratten, ${ }^{2}$ \\ David A. Spratt, ${ }^{2}$ and Michael Wilson ${ }^{2}$ \\ ${ }^{1}$ Department of Preventive Dentistry, Academic Centre for Dentistry Amsterdam (ACTA), \\ University of Amsterdam and VU University Amsterdam, Gustav Mahlerlaan 3004, 1081 LA Amsterdam, The Netherlands \\ ${ }^{2}$ Department of Microbial Diseases, UCL Eastman Dental Institute, 256 Gray's Inn Road, London WC1X 8LD, UK \\ ${ }^{3}$ Department of Drug Sciences, University of Pavia, Via Taramelli 12, 27100 Pavia, Italy \\ ${ }^{4}$ Microbiology Section, Department of Pathology and Diagnostics, University of Verona, Strada Le Grazie 8, 37134 Verona, Italy \\ ${ }^{5}$ DIPTERIS, University of Genoa, Corso Europa 26, 16132 Genoa, Italy \\ ${ }^{6}$ Department of Cariology, Institute of Odontology at Sahlgrenska Academy, University of Gothenburg, \\ P.O. Box 450, 40530 Göteborg, Sweden
}

Correspondence should be addressed to Egija Zaura, e.zaura@acta.nl

Received 14 June 2011; Accepted 14 July 2011

Academic Editor: Itzhak Ofek

Copyright (c) 2011 Egija Zaura et al. This is an open access article distributed under the Creative Commons Attribution License, which permits unrestricted use, distribution, and reproduction in any medium, provided the original work is properly cited.

The aim of the current study was to investigate the anticariogenic potential of the (sub)fractions obtained from the edible mushroom shiitake (Lentinula edodes) in in vitro caries model. We used a modified constant depth film fermentor (CDFF) with pooled saliva as the inoculum and bovine dentin as a substratum. The test compounds were low molecular weight fraction (MLMW) of the shiitake extract and subfractions 4 and 5 (SF4 and SF5) of this fraction. Chlorhexidine (CHX) and water served as a positive and a negative control, respectively. Dentin mineral loss was quantified (TMR), microbial shifts within the microcosms were determined (qPCR), and the acidogenicity of the microcosms was assessed (CIA). From the compounds tested, the SF4 of shiitake showed strong inhibiting effect on dentin demineralization and induced microbial shifts that could be associated with oral health. The acid producing potential was increased, suggesting uncoupling of the glycolysis of the microbiota by the exposure to $\mathrm{SF} 4$. In conclusion, the results suggest that SF4 of shiitake has an anticariogenic potential.

\section{Introduction}

Over the past decade, there has been an increasing number of food alerts creating a genuine crisis of confidence among consumers. Research on food safety and quality must therefore be a priority. To improve our understanding of the link between food and oral health, an international EU sixth framework program consortium project (NUTRIDENT, FOOD-CT-2006-36210) was granted with an overall aim to identify beverage/food constituents that are able to reduce the risk of two major dental diseases, caries and gingivitis.

Within the NUTRIDENT project, we have used the existing literature as a starting point for selecting foods or beverages that may contain such constituents. We have then built upon this knowledge by testing such materials for a range of biological activities that are relevant to the maintenance of oral health, that is, the prevention of caries and gingivitis. A number of high-throughput assays were designed and employed [1]. As a result of this work, we identified a low molecular mass extract $(<5,000 \mathrm{Da})$ of shiitake mushroom (Lentinula edodes) which has biological activities which, if displayed in vivo, could protect against dental caries. The most prominent observed biological activities of this extract, relevant to caries prevention, were (1) induction of the detachment of cariogenic microorganisms from hydroxyapatite, (2) changed cell surface hydrophobicity, (3) 
bactericidal activity against cariogenic microorganisms, (4) prevention of coaggregation of the microorganisms, and (5) disruption of signal transduction in Streptococcus mutans $[1,2]$. The extract from Lentinula edodes has been studied in rats, and an inhibitory effect on one of the virulence factors of S. mutans has been demonstrated: the extract inhibited water-insoluble glucan formation by glycosyltransferases of this organism [3]. There are few reports related to general antimicrobial effects of different compounds obtained from shiitake. Aqueous extract from $L$. edodes has shown high antimicrobial activity against food-borne pathogenic bacterial strains [4]. Furthermore, a diet containing 5\% of dried L. edodes consistently resulted in lower viable counts of total bacteria, Escherichia coli, streptococci, and lactic acidproducing bacteria in the intestinal microbiota of piglets [5].

Dental caries is a multifactorial disease with low $\mathrm{pH}$ as a driving force for mineral dissolution. We have developed an in vitro dental caries biofilm model $[6,7]$ which combines cariogenic microorganisms with dental hard tissue substratum (dentin or enamel) and allows modeling of frequent acid challenges by sucrose pulsing within a constant depth film fermentor (CDFF) [8]. The complexity of the model can be varied by selecting defined microbial consortia or saliva-derived microcosms as inocula $[8,9]$. Different output parameters, related to the cariogenic potential of the biofilms, can be assessed within this model system. The most relevant output for the anticariogenicity tests is mineral loss quantification [10]. Thus, any compound with anticariogenic activity claims should result in inhibited mineral loss in in vitro or in situ-tests or ultimately, in reduction of caries, in vivo. Other, the so-called surrogate output parameters include reduction of acid producing potential of the biofilms and microbial shifts towards healthassociated microorganisms.

The aim of the current study was to assess the effects of low molecular weight fraction of shiitake mushroom and two subfractions of this fraction on dentin demineralization, microbial composition, and acidogenic potential of salivaderived microcosms in our CDFF caries model.

\section{Materials and Methods}

2.1. Preparation of Fractions and Subfractions Obtained from Shiitake Mushroom Extract. The freeze-dried test compounds were obtained as described by Daglia et al. [2], reconstituted to the original $2 \mathrm{x}$ concentration in ultrapure (MilliQ, Millipore) sterile water and stored at $-20^{\circ} \mathrm{C}$ until used. Before each experiment, a frozen aliquot of the test compound was thawed and diluted 1:10 in Millipore grade sterile water. The treatment solutions contained $0.2 \mathrm{x}$ diluted low molecular weight fraction of shiitake mushroom (MLMW) extract, $0.2 \mathrm{x}$ diluted subfraction 4 of MLMW and $0.2 \mathrm{x}$ diluted subfraction 5 of MLMW.

2.2. Constant Depth Film Fermentor (CDFF) Experiments. Plaque microcosms were grown on dental hard tissue specimens in a modified CDFF model [7]. The modification

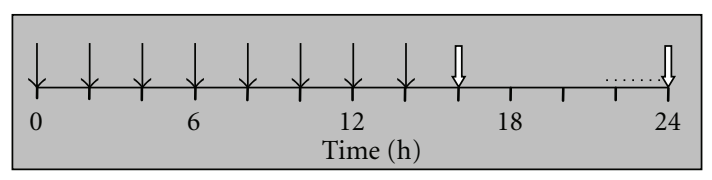

FIgURE 1: A diagram of daily CDFF regime for 8 days. On each day, a DMM supply (solid horizontal line) was interrupted by eight 2-hourly 5 min sucrose pulses (black arrows) and two $10 \mathrm{~min}$ treatments (white arrows). The treatments occurred $2 \mathrm{~h}$ after the last sucrose pulse and $20 \mathrm{~min}$ before the end of the "resting period" (DMM supply for $10 \mathrm{~h}$ ). Dashed horizontal line indicates the sampling time on day 8 .

of the CDFF involves operating it in a reciprocal mode: the turntable rotates 180 degrees back and forth, allowing two simultaneous growth conditions (two treatment modes) within one CDFF run. Sucrose pulses, media flow, and the treatments are provided by calibrated, computercontrolled peristaltic pumps (Type MS-4/6-100, Ismatec, Zürich, Switzerland) under software developed in LabView (National Instruments).

Coronal dentin from bovine incisors was cut into $5 \mathrm{~mm}$ diameter discs and was recessed into PTFE pans and assembled into the turntable of the CDFF. Plaque-enriched saliva from 10 healthy individuals (no use of antibiotics in the last 3 months) was collected and pooled at equal volumes, 10\% glycerol added and stored in $10 \mathrm{~mL}$ aliquots at $-80^{\circ} \mathrm{C}$. To inoculate each CDFF run, $9 \mathrm{~mL}$ of thawed pooled saliva was mixed with $200 \mathrm{~mL}$ defined mucin medium (DMM) [11]. The inoculum was pumped into the CDFF for $1.5 \mathrm{~h}$ (flow rate $2.3 \mathrm{~mL} / \mathrm{min}$ ) with the CDFF operated in the conventional mode $\left(360^{\circ}\right.$ rotation). One hour elapsed between the end of inoculation and the start of DMM flow. Then, DMM was supplied through two delivery inlets at $0.3 \mathrm{~mL} / \mathrm{min}$ per inlet with CDFF still operating in the conventional mode. The CDFF was operated at $37^{\circ} \mathrm{C}$ and under a continuous gas supply of $10 \% \mathrm{CO}_{2}$ and $10 \% \mathrm{H}_{2}$ in $\mathrm{N}_{2}$ at a flow rate of $50 \mathrm{~mL} / \mathrm{min} \pm 0.5 \mathrm{~mL} / \mathrm{min}$.

After $24 \mathrm{~h}$, the CDFF was switched to the reciprocal mode ( $180^{\circ}$ oscillation) and the first $10 \mathrm{~min}$ treatment was started. Sterile water was used as a negative control of the treatment on one side of each CDFF run, while positive control $(0.12 \%$ chlorhexidine digluconate solution $)$ or one of the test solutions was applied on the opposite side of the CDFF. After a 10 min resting period, the LabView program with daily treatment regimen was started. The cariogenic potential of the microcosm was modelled by frequent (eight 2-hourly 5 minute pulses/day, flow rate $1.2 \mathrm{~mL} / \mathrm{min} /$ inlet) pulses with $10 \%(\mathrm{w} / \mathrm{v})$ sucrose solution. "Night" or remineralization phase was simulated by daily $10 \mathrm{~h}$ period of DMM supply alone. Summary of the daily regimen is shown in Figure 1. Two $10 \mathrm{~min}$ treatments were given each day at a rate of $1.0 \mathrm{~mL} / \mathrm{min}$. The first treatment commenced $2 \mathrm{~h}$ after the last sucrose pulse, while the second treatment started $20 \mathrm{~min}$ before the end of the "Night" period.

Two independent CDFF runs per compound (three test compounds and one positive control) were performed. 

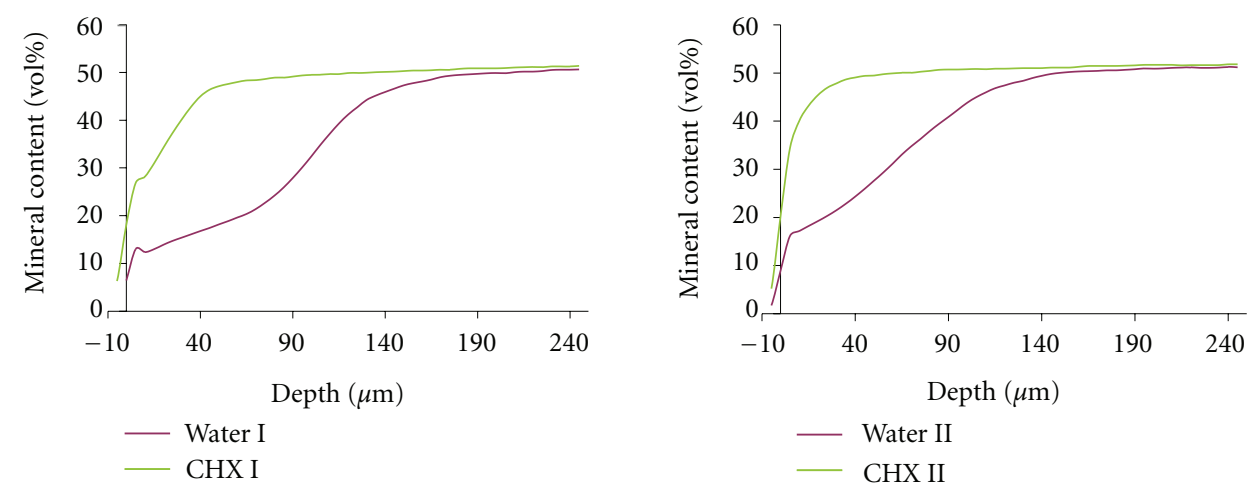

(a)
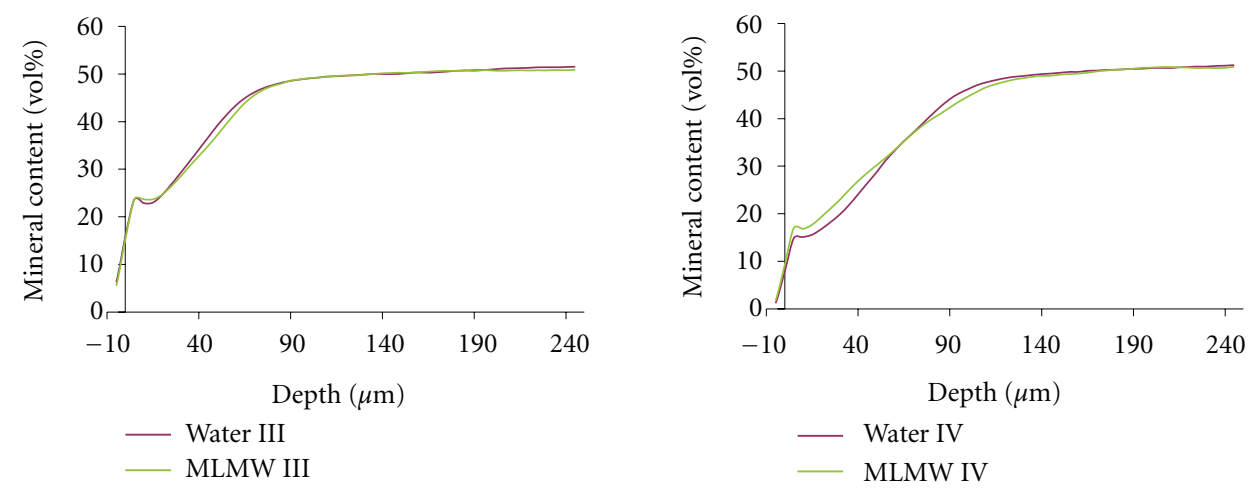

(b)
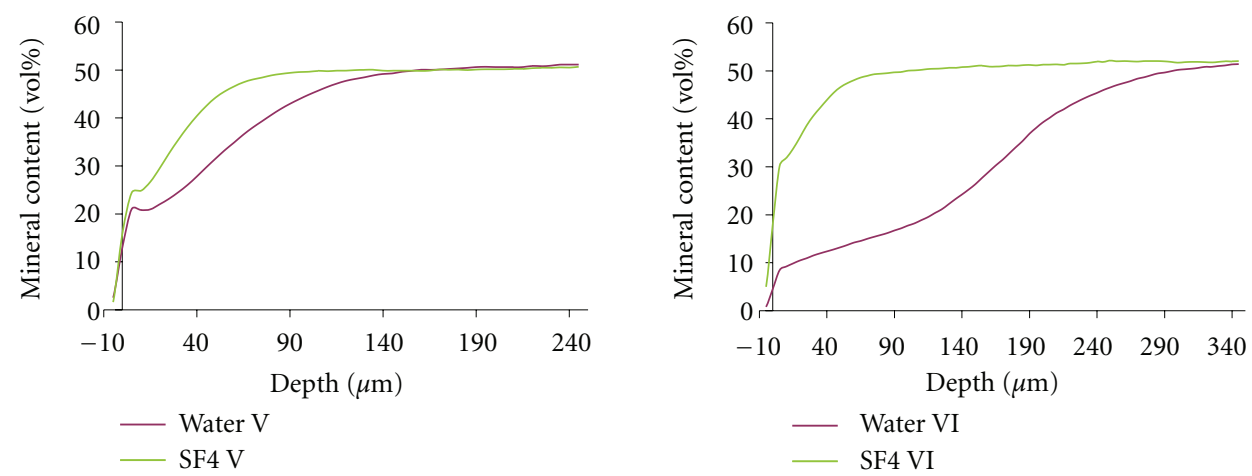

(c)
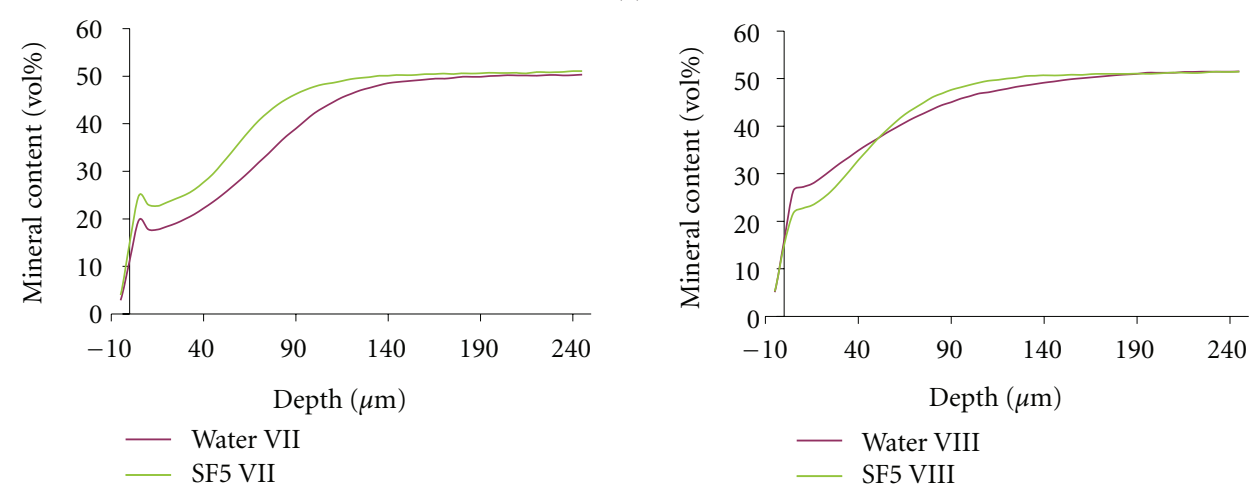

(d)

FigURE 2: Average mineral content profiles showing the extent of demineralization of dentin after microcosm growth for 8 days in constant depth film fermentor (CDFF) per CDFF run (Roman numbers I-VIII-eight CDFF runs) and per treatment: (a) chlorhexidine digluconate (CHX), (b) mushroom low molecular weight fraction (MLMW), (c) subfraction nr 4 (SF4), and (d) Subfraction nr 5 (SF5) of the MLMW fraction of shiitake extract. The two graphs per treatment are the profiles obtained from the duplicate CDFF runs of the respective treatment. Each profile is an average of the transversal microradiography images obtained from 10 dentin specimens. 


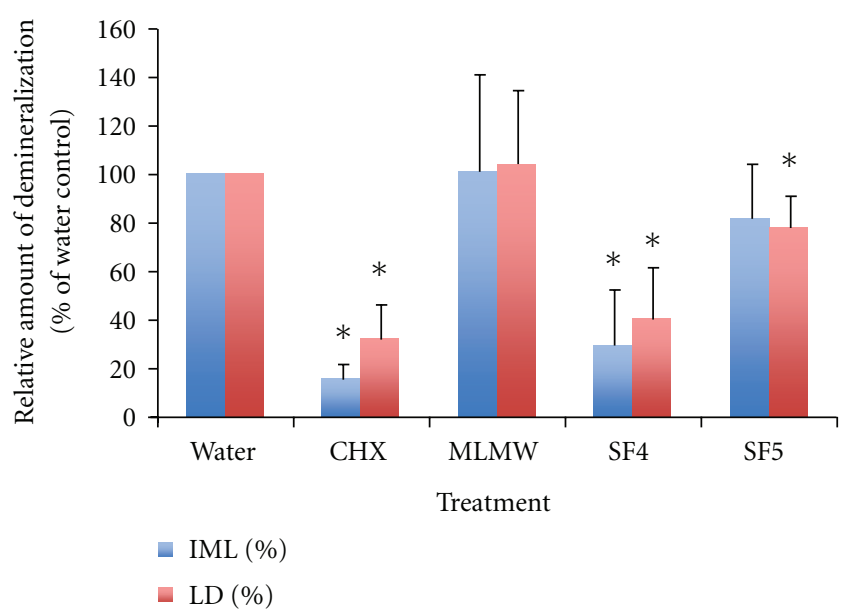

FIGURE 3: Average amount of demineralization (integrated mineral loss, IML, and lesion depth, LD) relative to the respective water control of each treatment (IML and LD of the water group were set to $100 \%$ to normalize the data among different CDFF runs). $\mathrm{CHX}-$ chlorhexidine digluconate; MLMW-mushroom low molecular weight fraction; SF4 - subfraction nr 4 of MLMW fraction; SF5subfraction nr 5 of MLMW fraction. $N=20$ samples per treatment. Error bars indicate standard deviation. ${ }^{*}$ One sample $t$ test, treatment versus $100 \%$ (water control), significantly different at $P<0.001$.

2.3. Sampling and Sample Processing. On day 8, two CDFF sample pans per each treatment group were removed, resulting in 10 samples per treatment group. Sampling occurred during the resting period, 7.5-9.5 h after the last sucrose pulse and 5.5-7.5 h after the last treatment (dashed line in Figure 1).

Immediately after the retrieval from the CDFF, the biofilm was removed from the dentin surface by scraping against the lid of an Eppendorf tube. Subsequently, the biofilm was centrifuged for $30 \mathrm{~s}$ at $16,060 \times \mathrm{g}$ and either $1 \mathrm{~mL}$ $1 \%$ glucose in buffered peptone water (BPW) solution (fermenting plaque) or BPW solution alone (resting plaque) was added to the biofilm and incubated at $37^{\circ} \mathrm{C}$ for $30 \mathrm{~min}$. After that, the vials were cooled on ice. The samples were heated at $80^{\circ} \mathrm{C}$ for 5 minutes and again cooled on ice [12]. The vials were centrifuged at $16,060 \times \mathrm{g}$ for 15 minutes at $4^{\circ} \mathrm{C}$. From the supernatant, $200 \mu \mathrm{L}$ were transferred into a vial with a microspin filter (Ultrafree-MC $0.22 \mu \mathrm{m}$, Millipore, Bedford, Mass, USA) and centrifuged at $13,684 \times \mathrm{g}$ for 5 minutes at $4^{\circ} \mathrm{C}$; the remaining supernatant was discarded. The filtered supernatants for organic acid determination and the pellets for protein analysis were stored at $-80^{\circ} \mathrm{C}$ until further analyses. The dentin discs were stored at $4^{\circ} \mathrm{C}$ for the assessment of mineral loss by transverse microradiography (TMR).

Organic acids were determined as their anions by capillary electrophoresis on the Waters Capillary Ion Analyzer (Milford, Mass, USA) [13]. Sodium salts of formic, acetic, propionic, butyric, succinic, and lactic acids were used to prepare single and mixed standard solutions in ultrapure water (for calibration curves for each acid separately). Oxalic acid was included in all samples as an internal standard. Formic, butyric, succinic, propionic, acetic, and lactic acid were determined in duplicate samples.

The amount of acid was normalized by amount of protein/sample. The protein amount was determined by Bradford protein analysis method [14]. Results were expressed as $\mu \mathrm{g}$ protein/sample.

Dentin discs were sectioned and processed for transverse microradiography (TMR) as described elsewhere [15]. In brief, $200 \mu \mathrm{m}$ thin dentin sections were radiographed together with an aluminium stepwedge on a high-resolution film with a nickel-filtered $\mathrm{Cu}-\mathrm{K} \alpha$ source. The radiographic image was analyzed with a microscope-videocameramicrocomputer setup and dedicated software (TMR 2000, version 2.0.27.13, Inspektor Research Systems, Amsterdam, The Netherlands). Data obtained were the mineral content profiles of the lesions, lesion depth, and the total amount of mineral removed (integrated mineral loss).

The numbers of Streptococcus sanguinis, Streptococcus mutans, Lactobacillus casei, Veillonella dispar, Neisseria subflava, Actinomyces naeslundii, Prevotella intermedia, Fusobacterium nucleatum, and total bacterial $16 \mathrm{~S}$ rDNA were determined by using multiplex quantitative PCR (qPCR) [16]. In brief, DNA was extracted from plaque biofilms using a phenol:chloroform: iso-amyl alcohol $(25: 24: 1)$ beadbeating extraction method [17], which involves physical cell lysis, protein removal, and finally DNA precipitation using polyethylene glycol. Three triplex qPCR assays were then carried out using $2 \mu \mathrm{L}$ extracted DNA to enumerate eight oral taxa as well as the total number of organisms. The assays were performed using the Rotor-Gene 6500 (QIAGEN) instrument and Sensimix Probe (Bioline) qPCR mix according to manufacturer's instructions using previously published oligonucleotide sequences [16].

2.4. Statistical Analyses. The effects of the treatments on dentin demineralization (integrated mineral loss, IML, and lesion depth, LD), biomass (protein amount), and acidogenicity of biofilms (acetate, lactate, propionate, succinate, butyrate, and formate) were assessed by independent samples $t$-test and by one-sample $t$-test, where the data in each treatment group was calculated as a relative proportion of the average value from the respective water group (negative control), set at $100 \%$. The qPCR data were log transformed and used as absolute values and as a proportion of the total counts (universal probe counts) in statistical comparisons between the treatment groups and their respective controls, using Mann-Whitney $U$ test. All tests were performed in SPSS, version 17.0. Significance level was set at 0.05 .

\section{Results and Discussion}

In this study, we grew saliva-derived microcosms at the conditions that mimicked a cariogenic situation in vivo (eight daily sucrose pulses). We also allowed recovery of the microbial ecosystem during a daily resting period of $10 \mathrm{~h}$. This in turn mimicked a night period in vivo. Biofilms grown in all eight constant depth film fermentor (CDFF) runs 


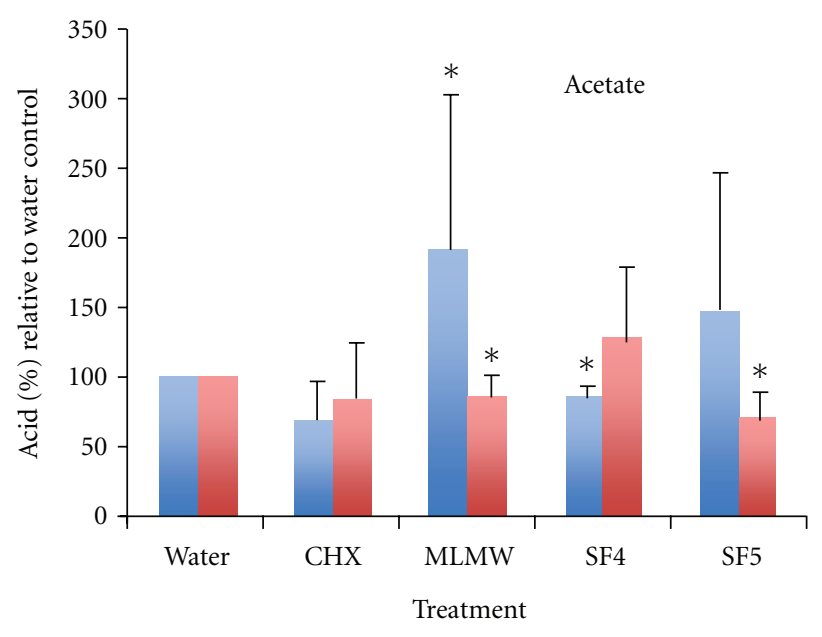

(a)

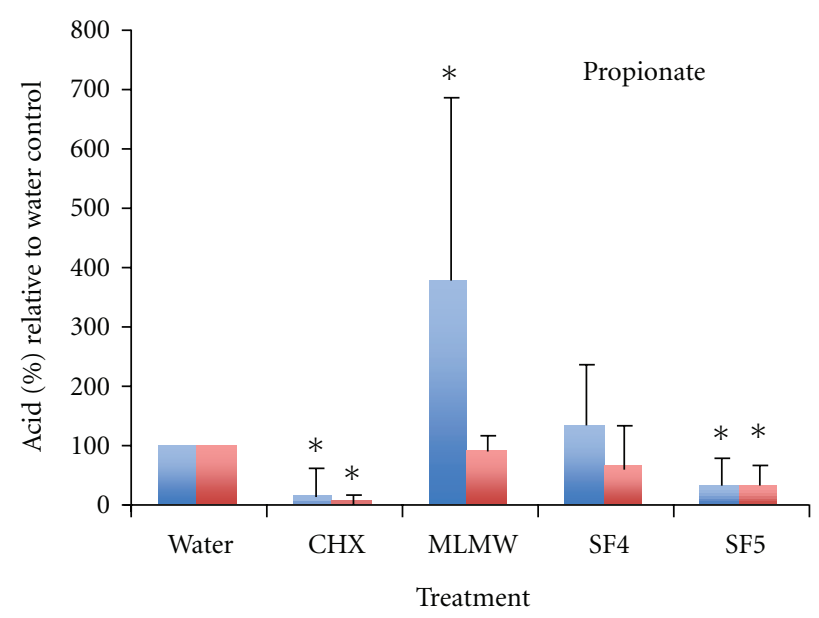

(c)

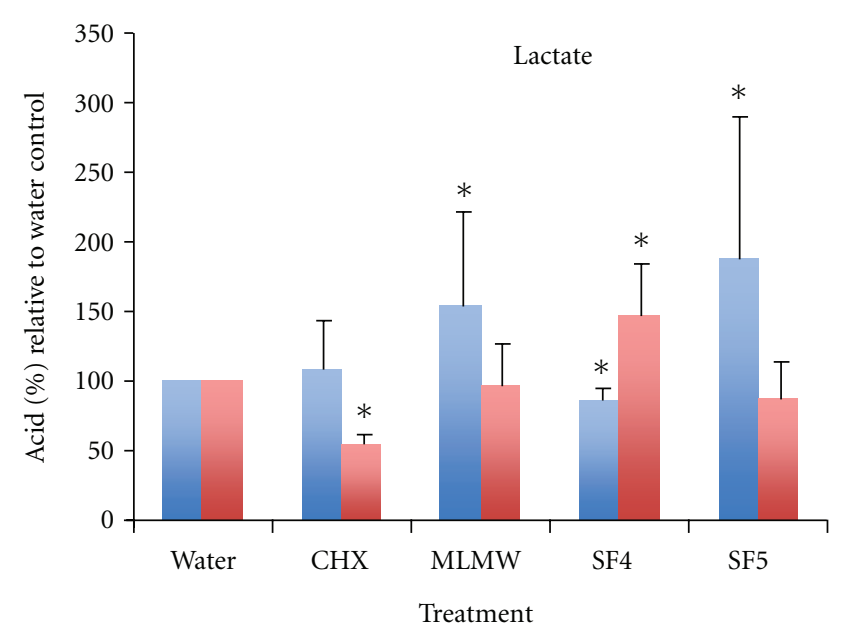

(b)

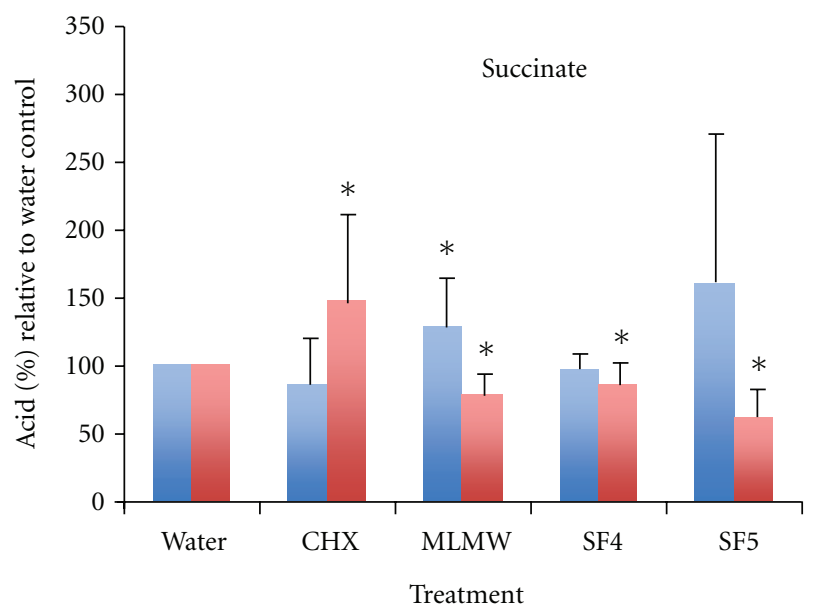

(d)

FIgURE 4: Amount of (a) acetate, (b) lactate, (c) propionate, and (d) succinate in 8-day microcosms at a resting state (blue bars) and at a fermenting state after 30 min incubation with $1 \%$ glucose (red bars) in the treatment group samples (CHX-chlorhexidine digluconate; MLMW-mushroom low molecular weight extract; SF4—subfraction nr 4 of MLMW extract; SF5—subfraction nr 5 of MLMW extract) relative to the samples exposed to water (negative control). Treatments were performed twice daily for 10 min (Figure 1 ). ${ }^{*}$ One sample $t$-test, treatment versus $100 \%$ (water control), significantly different at $P<0.001$.

resulted in demineralization of the underlying substratumbovine dentin (Figure 2 and Table 1). There was a large variation among the individual CDFF runs in the amount of mineral loss and the depth of the lesions (red mineral profiles in Figure 2 represent eight individual CDFF runs (I-VIII) exposed to water). This underlines the difficulty of controlling the complex ecological systems such as microcosms derived from natural sources [18]. The reciprocal mode of the CDFF (adaptation to the CDFF with back-andforth movement of the rotating pane [7]) allowed within each individual run the growth of both, the treated and the negative control (water) exposed biofilms. This provided the internal control to each of the treatment groups, allowing statistical comparisons between the matched test and control samples.

Twice daily exposure to the positive control, $0.12 \%$ chlorhexidine digluconate (CHX), had pronounced inhibi- tory effect on the cariogenic potential of the microcosms. This was seen as statistically significantly inhibited dentin demineralization (average mineral content profiles in Figure 2; IML and LD in Figure 3, Table 1), biomass (protein amount in Table 1), and main organic acids (Table 1), as well as significantly reduced absolute microbial counts (Table 2) compared to the water-exposed samples. CHX is a broadspectrum antimicrobial agent that has been proven to have clinical antiplaque and antigingivitis effects [19] and has been shown to inhibit acids in resting and fermenting plaque $[20,21]$. The previous reports on CDFF-grown biofilms treated with CHX range from little or some effects on biofilm viability and composition $[22,23]$ to nearly complete inhibition of the biofilm [7], depending on the type and complexity of the inoculum, growth medium, substratum, and many other parameters, such as the exposure time and the clearance of the antimicrobial from the system. Our 
results with $\mathrm{CHX}$ showed that the conditions we have chosen were appropriate to use for the series of experiments with the test compounds that were selected in the high-throughput tests [1].

The test compounds that we have tested were derived from natural edible mushroom shiitake, Lentinula edodes, as described by Daglia et al. [2]. Using our CDFF model, we tested the anticariogenic potential of the low molecular weight fraction of the mushroom extract (MLMW) and the two subfractions from this fraction-subfraction 4 (SF4) and subfraction 5 (SF5). Among the three test compounds, the SF4 showed the strongest anticariogenic potential. The twice daily treatment with SF4 highly inhibited demineralization of dentin, resulting in significantly reduced IML and LD compared to the respective water control samples (Figures 2 and 3 and Table 1). The treatment with SF5 showed some, though still statistically significant, reduction of lesion depth, LD, while the treatment with MLMW did not have any significant effects on the demineralization of dentin (Figure 3 and Table 1). The average mineral content profiles (Figure 2) showed that only one of the two replicate experiments with SF5 (CDFF VII but not CDFF VIII) resulted in slight inhibition of demineralization of dentin, rendering the results of SF5 inconclusive.

Unlike CHX, none of the test compounds affected the biomass of the microcosms, that is, there were no significant differences in either protein amount (Table 1) or universal $16 \mathrm{~S}$ rDNA probe counts (Table 2) between the treated and the respective water-control samples. However, the microbial community composition in the SF4-exposed samples was significantly changed, compared to the watercontrol samples. SF4 significantly increased absolute counts of Fusobacterium nucleatum and Neisseria subflava (Table 2), and the relative proportions of these two microorganisms, together with the relative proportions of Veillonella dispar and Actinomyces naeslundii (Figure 5). At the same time, the proportion of the other microorganisms that were not targeted in this study, but were quantified as the difference between the universal $16 \mathrm{~S}$ rDNA probe counts and the sum of the targeted species, significantly decreased. Thus, the effects of the SF4 were beyond the selected targeted species for this study and have affected other microorganisms as well. The MLMW-exposed biofilms showed statistically significantly increased absolute counts of F. nucleatum, while there were no significant effects on the proportions of different microorganisms induced by this compound. The SF5-exposed biofilms showed shifts in microbial community composition similar to the shifts induced by SF4; however, none of these shifts reached statistical significance (Figure 5). Both, veillonellae and neisseriae are microorganisms associated with oral health [24], while fusobacteria are a part of noncariogenic resident oral flora, also known as a bridging organism in maturing dental biofilm [25]. Increase in amount and/or proportions of these noncariogenic microorganisms under highly cariogenic conditions in our CDFF microcosms indicates occurrence of health-associated microbial shifts due to the exposure to the shiitake-derived test compounds.

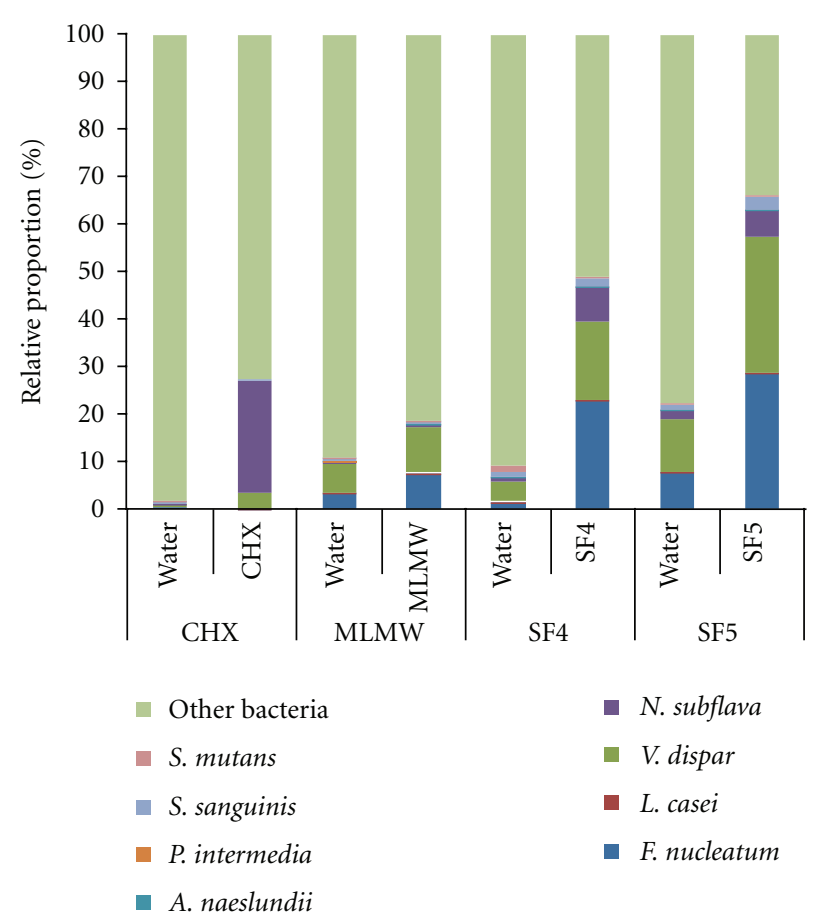

FIGURE 5: Relative proportions of microorganisms in microcosm samples. The other bacteria were calculated as the difference between the universal probe counts and the sum of the 8 targeted probe counts. The data are average from 6 samples obtained in 2 CDFF runs per treatment. CHX-chlorhexidine digluconate; MLMW-mushroom low molecular weight fraction; SF4 - subfraction nr 4 of MLMW fraction; SF5—subfraction nr 5 of MLMW fraction.

There were no strong and conclusive inhibitory effects of the test compounds on the acidogenicity of the microcosms observed. SF4 showed some inhibitory activity on biofilms at the resting state: there was significantly less acetate (Figure 4) and lactate (Table 1, Figure 4) in the SF4-exposed resting biofilms compared to the water-exposed biofilms. In contrary, the glucose-fermenting SF4-exposed biofilms produced significantly more lactate than their respective water controls (Table 1 and Figure 4). Similar effects were observed in high-throughput acidogenicity assays with single species biofilms of S. mutans [1]. The SF5-exposed biofilms produced less propionate in the resting and the fermenting biofilms, and less acetate and succinate in the fermenting samples, while the resting samples showed significantly more lactate compared to the water control (Table 1 and Figure 4). The MLMW-exposed resting state biofilms had increased amounts of all major acid anions measured, including lactate, and reduced amounts of acetate and succinate in the fermenting samples. Increased lactate production during the 30 minute incubation with glucose suggests enhanced acidogenic potential of the biofilms previously exposed to the test compounds. This however did not result in increased cariogenic activity (demineralization of the dentin substratum in our CDFF model). In contrary, exposure to SF4 strongly inhibited demineralization (Figures 2 and 3). The reasons for the increased lactate production are not 


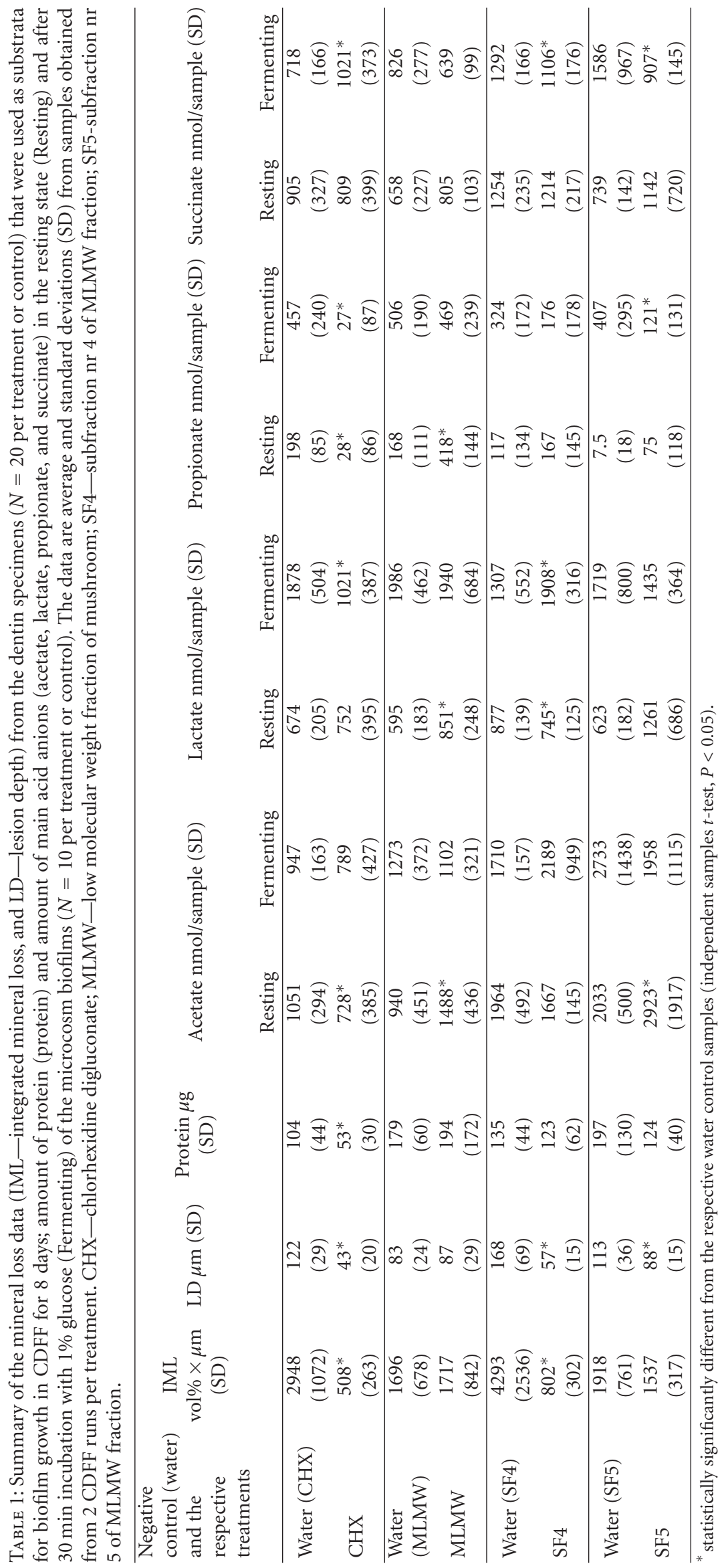




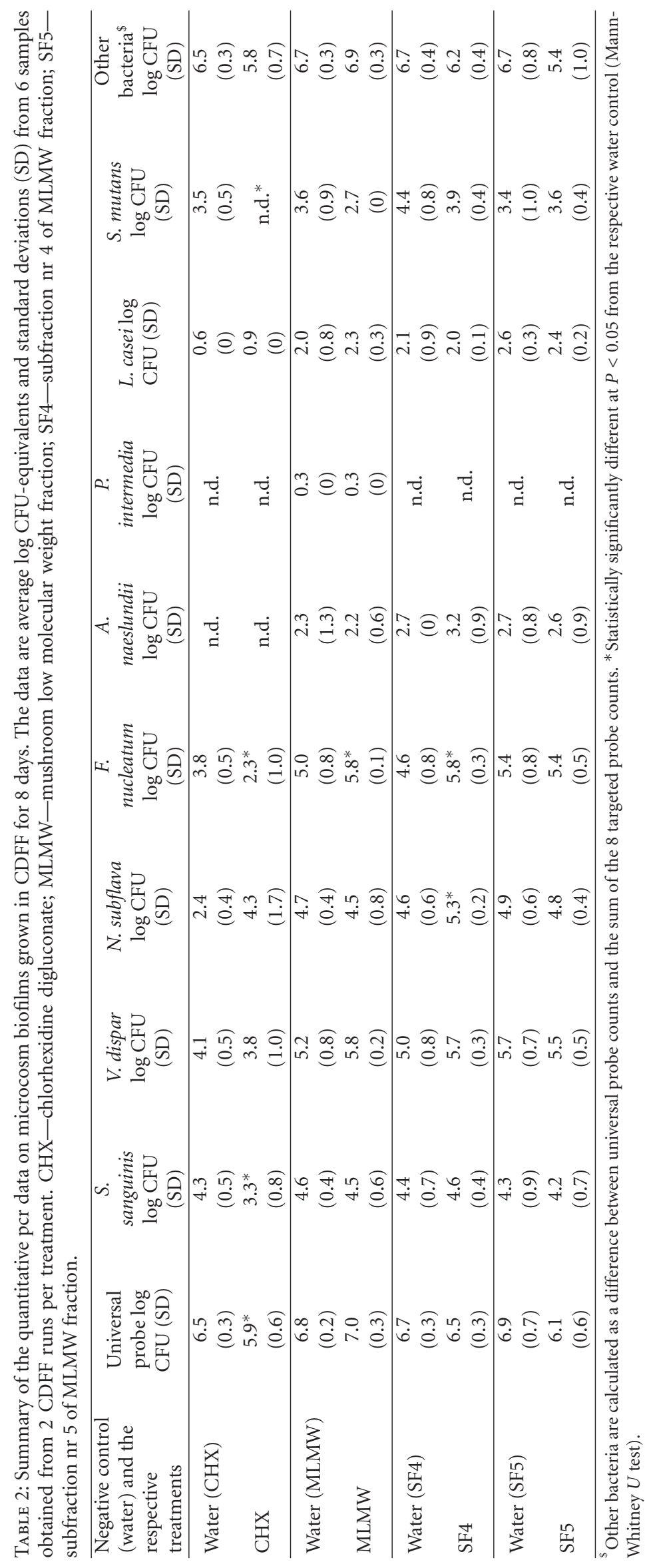


known and should be investigated further. It might be related to a phenomenon known as uncoupling $[26,27]$, where glycolysis is uncoupled from biomass production and enters a futile cycle. In lactic acid bacteria used in dairy industry, uncoupling has been shown to be triggered by stress factors such as subbactericidal concentrations of antimicrobials or elevated temperature [28] and leads to less efficient energy usage. Our results suggest that the subbactericidal concentrations of the SF4 of shiitake mushroom may have induced uncoupling in the biofilm cells of the plaque microcosms.

Based on the results above, we can conclude that the subfraction 4 (SF4) of the low molecular weight fraction of the shiitake mushroom has strong anticariogenic potential. This anticariogenic potential of SF4 is most likely contributable to the observed changes in microbial composition and inefficient energy usage due to uncoupling of the glycolysis.

\section{Acknowledgment}

The research leading to these results has received funding from the European Union's Sixth Framework Programme (FP6) under the contract FOOD-CT-2006-036210 (Project NUTRIDENT).

\section{References}

[1] D. A. Spratt et al., "Evaluation of plant and fungal extracts for their anti-gingivitis and anti-caries activity," Journal of Biomedicine and Biotechnology. In press.

[2] M. Daglia et al., "Vegetable food components with potential activity on the development of microbial oral diseases," Journal of Biomedicine and Biotechnology. In press.

[3] N. Shouji, K. Takada, K. Fukushima, and M. Hirasawa, "Anticaries effect of a component from shiitake (an Edible Mushroom)," Caries Research, vol. 34, no. 1, pp. 94-98, 2000.

[4] M. E. Venturini, C. S. Rivera, C. Gonzalez, and D. Blanco, "Antimicrobial activity of extracts of edible wild and cultivated mushrooms against foodborne bacterial strains," Journal of Food Protection, vol. 71, no. 8, pp. 1701-1706, 2008.

[5] C. J. van Nevel, J. A. Decuypere, N. Dierick, and K. Molly, “The influence of lentinus edodes (Shiitake mushroom) preparations on bacteriological and morphological aspects of the small intestine in piglets," Archives of Animal Nutrition, vol. 57, no. 6, pp. 399-412, 2003.

[6] D. M. Deng and J. M. ten Cate, "Demineralization of dentin by Streptococcus mutans biofilms grown in the constant depth film fermentor," Caries Research, vol. 38, no. 1, pp. 54-61, 2004.

[7] D. M. Deng, C. van Loveren, and J. M. ten Cate, "Cariespreventive agents induce remineralization of dentin in a biofilm model," Caries Research, vol. 39, no. 3, pp. 216-223, 2005.

[8] J. Pratten, "Growing oral biofilms in a constant depth film fermentor (CDFF)," Current Protocols in Microbiology, vol. 6, Unit 1B.5.1-1B.5.18, 2007.

[9] A. J. McBain, R. G. Bartolo, C. E. Catrenich, D. Charbonneau, R. G. Ledder, and P. Gilbert, "Growth and molecular characterization of dental plaque microcosms," Journal of Applied Microbiology, vol. 94, no. 4, pp. 655-664, 2003.
[10] M. D. Lagerweij, E. de Josselin de Jong, and J. M. ten Cate, "The video camera compared with the densitometer as a scanning device for microradiography," Caries Research, vol. 28, no. 5, pp. 353-362, 1994.

[11] L. Wong and C. H. Sissons, "A comparison of human dental plaque microcosm biofilms grown in an undefined medium and a chemically defined artificial saliva," Archives of Oral Biology, vol. 46, no. 6, pp. 477-486, 2001.

[12] V. A. M. Gerardu, C. van Loveren, M. Heijnsbroek, M. J. Buijs, G. A. van der Weijden, and J. M. ten Cate, "Effects of various rinsing protocols after the use of amine fluoride/stannous fluoride toothpaste on the acid production of dental plaque and tongue flora," Caries Research, vol. 40, no. 3, pp. 245-250, 2006.

[13] J. J. M. Damen, M. J. Buijs, and J. M. ten Cate, "Acidogenicity of buccal plaque after a single rinse with amine fluoridestannous fluoride mouthrinse solution," Caries Research, vol. 36, no. 1, pp. 53-57, 2002.

[14] M. M. Bradford, "A rapid and sensitive method for the quantitation of microgram quantities of protein utilizing the principle of protein dye binding," Analytical Biochemistry, vol. 72, no. 1-2, pp. 248-254, 1976.

[15] E. Zaura, M. J. Buijs, and J. M. ten Cate, "Effects of ozone and sodium hypochlorite on caries-like lesions in dentin," Caries Research, vol. 41, no. 6, pp. 489-492, 2007.

[16] L. Ciric, J. Pratten, M. Wilson, and D. Spratt, “Development of a novel multi-triplex qPCR method for the assessment of bacterial community structure in oral populations," Environmental Microbiology Reports, vol. 2, no. 6, pp. 770-774, 2010.

[17] R. I. Griffiths, A. S. Whiteley, A. G. O’Donnell, and M. J. Bailey, "Rapid method for coextraction of DNA and RNA from natural environments for analysis of ribosomal DNAand rRNA-based microbial community composition," Applied and Environmental Microbiology, vol. 66, no. 12, pp. 54885491, 2000.

[18] J. W. Wimpenny, "Introduction," in Handbook of Laboratory Model Systems for Microbial Ecosystems, J. W. Wimpenny, Ed., CRC Press, Boca Raton, Fla, USA, 1988.

[19] P. D. Marsh, "Antimicrobial strategies in the prevention of dental caries," Caries Research, vol. 27, pp. 72-76, 1993.

[20] R. V. Oppermann, "Effect of chlorhexidine on acidogenicity of dental plaque in vivo," Scandinavian Journal of Dental Research, vol. 87, no. 4, pp. 302-308, 1979.

[21] J. S. van der Hoeven, D. Cummins, M. J. Schaeken, and F. J. van der Ouderaa, "The effect of chlorhexidine and zinc/triclosan mouthrinses on the production of acids in dental plaque," Caries Research, vol. 27, no. 4, pp. 298-302, 1993.

[22] J. Pratten, P. Barnett, and M. Wilson, "Composition and susceptibility to chlorhexidine of multispecies biofilms of oral bacteria," Applied and Environmental Microbiology, vol. 64, no. 9, pp. 3515-3519, 1998.

[23] M. Wilson, H. Patel, and J. H. Noar, "Effect of chlorhexidine on multi-species biofilms," Current Microbiology, vol. 36, no. 1, pp. 13-18, 1998.

[24] P. Marsh and M. V. Martin, "The resident oral microflora," in Oral Microbiology, P. Marsh and M. V. Martin, Eds., pp. 24-44, Elsevier, New York, NY, USA, 5th edition, 2009.

[25] P. E. Kolenbrander, R. N. Andersen, D. S. Blehert, P. G. Egland, J. S. Foster, and R. J. Palmer, "Communication among oral bacteria," Microbiology and Molecular Biology Reviews, vol. 66, no. 3, pp. 486-505, 2002.

[26] R. Otto, "Uncoupling of growth and acid production in Streptococcus cremoris," Archives of Microbiology, vol. 140, no. 2-3, pp. 225-230, 1984. 
[27] J. C. Senez, "Some considerations on the energetics of bacterial growth," Bacteriological Reviews, vol. 26, pp. 95-107, 1962.

[28] K. W. Turner and T. D. Thomas, "Uncoupling of growth and acid production in lactic streptococci," New Zealand Journal od Dairy Science and Technology, vol. 10, pp. 162-167, 1976. 

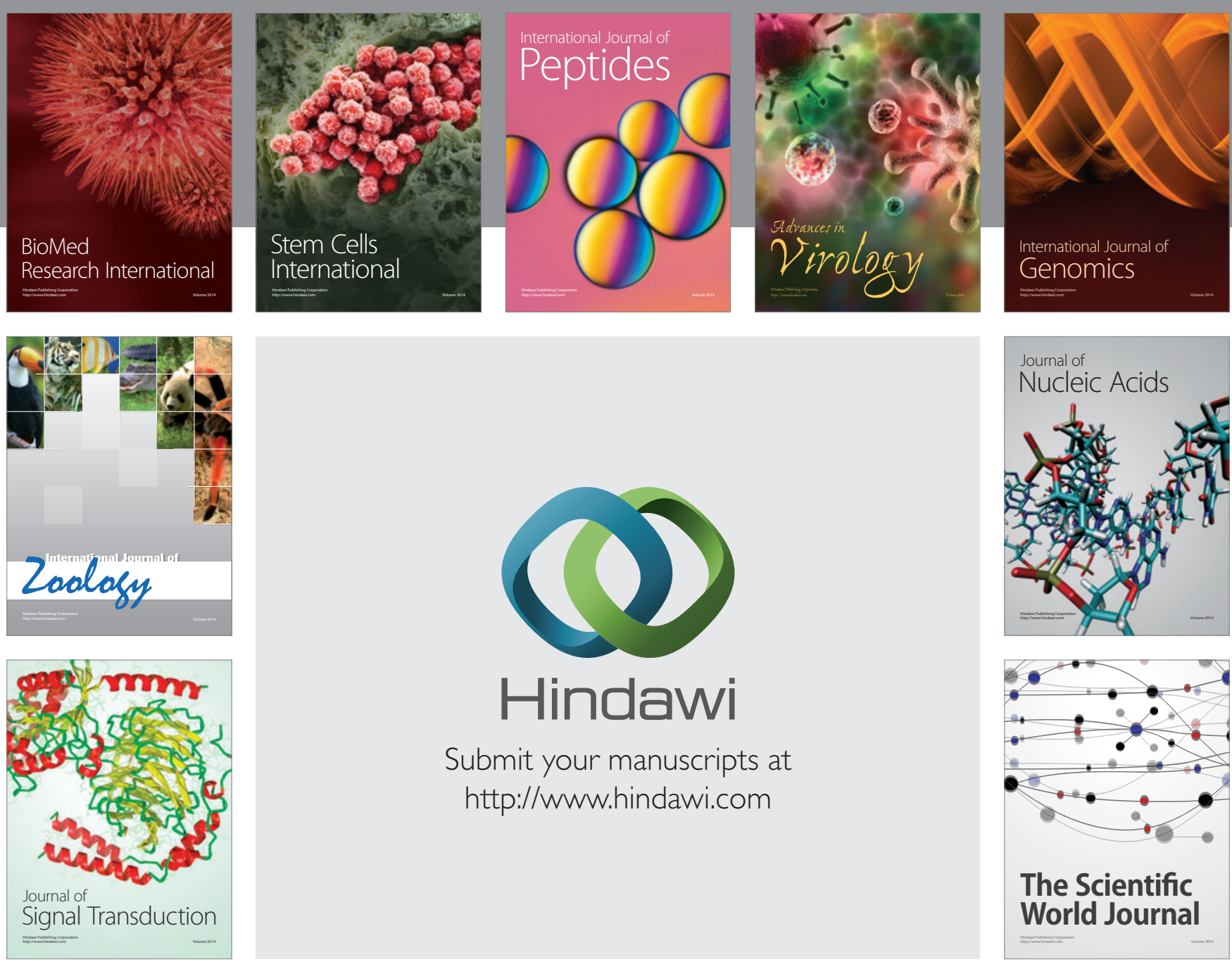

Submit your manuscripts at

http://www.hindawi.com
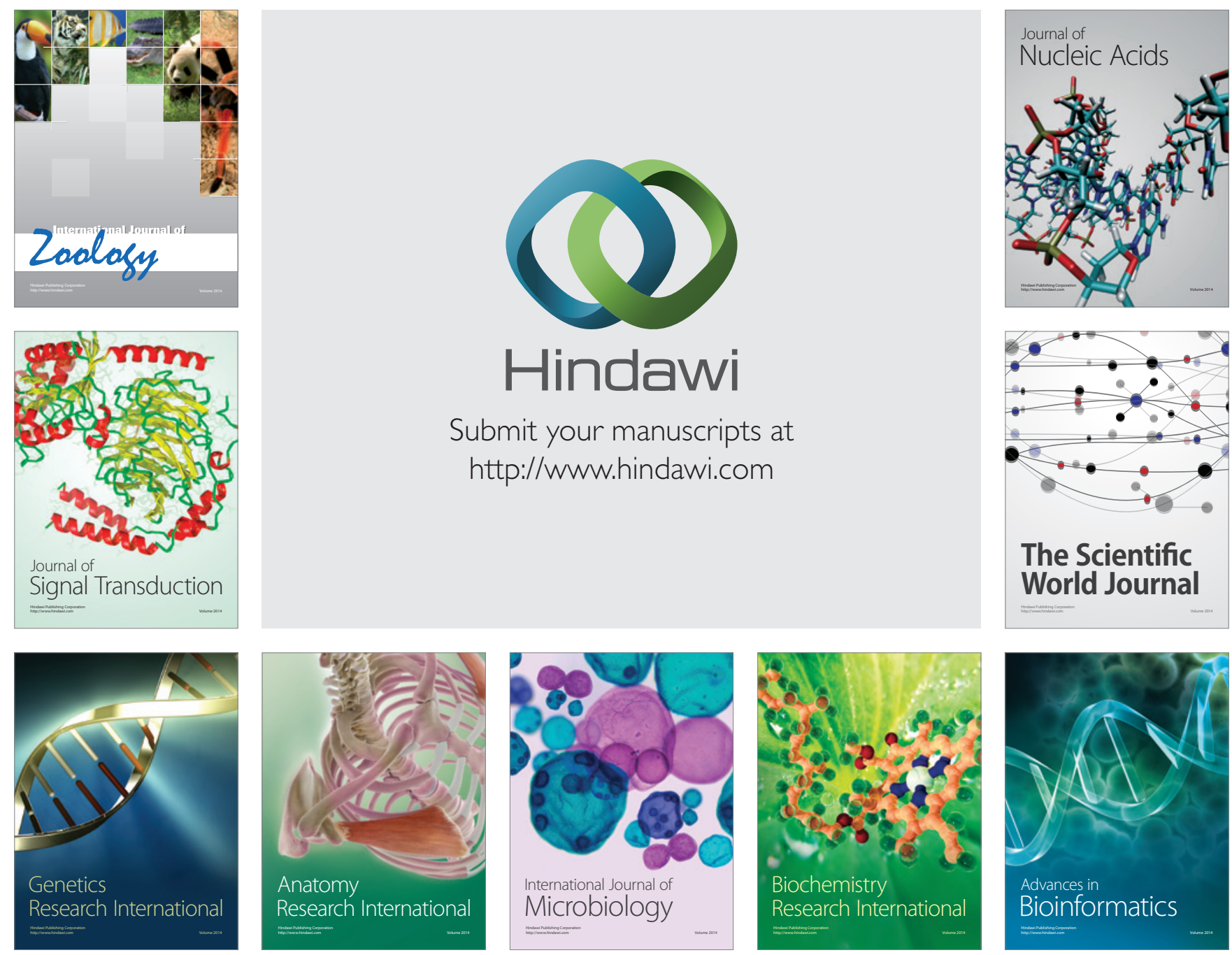

The Scientific World Journal
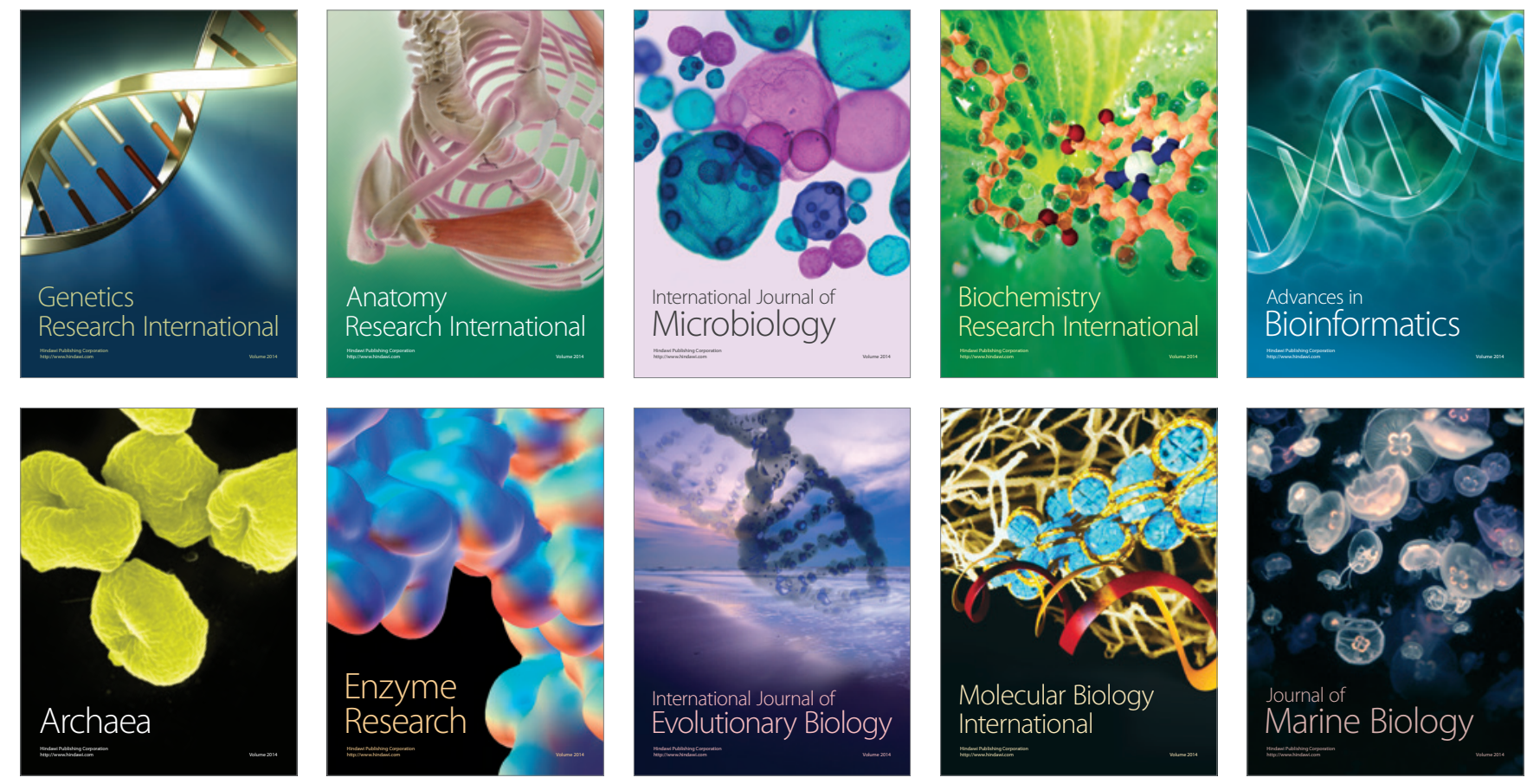